\title{
Validation of the multiresidual GC-MS method for determining plant protection product residues in strawberries
}

\author{
Helena BAŠA ČESNIK ${ }^{1 *}$
}

Received February 12, 2018; accepted September 23, 2018.

Delo je prispelo 12. februarja 2018, sprejeto 23. septembra 2018.

\begin{abstract}
Gas chromatography coupled with mass spectrometry was used for the introduction and validation of the multiresidual method for determining of plant protection product residues in strawberries. During the validation procedure, limits of quantification were set and the method was checked for its recovery, linearity, repeatability, reproducibility and measurement uncertainty. An interlaboratory comparison was also performed to check the accuracy of the method. The method was proven to be fit for purpose. Afterwards 19 strawberry samples were analysed for the presence of plant protection product residues using the validated method. In the strawberries 5 active substances, all fungicides, were found: chlorothalonil, cyprodinil, fludioxonil, metalaxyl+metalaxyl$\mathrm{M}$ and pyrimethanil. Residues of these active substances were in range $0.01-0.44 \mathrm{mg} / \mathrm{kg}$. No cases exceeding the maximum residue levels were measured.
\end{abstract}

Key words: pesticide residues; GC-MS; strawberries; plant protection product residues; multiresidual method
IZVLEČEK

\section{VALIDACIJA MULTIREZIDUALNE GC-MS METODE ZA DOLOČEVANJE OSTANKOV FITOFARMACEVTSKIH SREDSTEV V JAGODAH}

Plinsko kromatografijo sklopljeno $\mathrm{z}$ masno spektrometrijo smo uporabili za vpeljavo in validacijo multirezidualne metode za določanje ostankov fitofarmacevtskih sredstev $\mathrm{v}$ jagodah. Med validacijo smo postavili meje kvantitativne določitve metode in preverili izkoristek, linearnost, ponovljivost, obnovljivost in merilno negotovost metode. Sodelovali smo tudi $\mathrm{v}$ medlaboratorijski primerjavi, da smo preverili točnost metode. Za metodo se je izkazalo, da ustreza namenu. Nato smo z validirano metodo ugotavljali prisotnost ostankov fitofarmacevtskih sredstev v 19 vzorcih jagod. V njih smo določili 5 aktivnih spojin: klorotalonil, ciprodinil, fludioksonil, meatalaksil + metalaksil-M in pirimetanil. Ostanki teh aktivnih snovi so se gibali $\mathrm{v}$ območju $0,01-$ $0,44 \mathrm{mg} / \mathrm{kg}$. Preseganja maksimalnih dovoljenih količin ostankov nismo izmerili.

Ključne besede: ostanki pesticidov; GC-MS; jagode; ostanki fitofarmacevtskih sredstev; multirezidualna metoda

\section{INTRODUCTION}

Fruit is an important part of our diet for its nutrition and health properties. To prevent the destruction of food crops by agricultural pests and to improve plant quality, plant protection products (PPPs) must be used in fruit production. While monitoring the PPP residues in fruit, vegetables and cereals, we noticed (Baša Česnik et al., 2009) that fruit contains the highest number of active compounds. Farmers need to protect fruit against rot, mould and insects, otherwise the fruit would not grow. Strawberries are mainly attacked by the diseases Botrytis cinerea (Persoon), Colletotrichum acutatum (J.H. Simmonds), Oidium fragariae (Harz) and Mycospharella fragariae ((Tul.) Lindau) and by the pests Steneotarsonemus fragariae (Banks, 1901), Anthonomus rubi (Herbst, 1795), and Tetranychus urticae (C. L. Koch, 1836) (Sójka et al., 2015). Therefore, the use of PPPs during strawberry growth is inevitable.

Unfortunately, PPP residues can have a negative impact on consumer health when they exceed the Maximum Residue Levels (MRLs). Therefore, the monitoring of PPP residues is necessary. For proper monitoring, efficient analytical methods are required, which enable analysis of large number of active substances and their residues at the same time.

\footnotetext{
1 Agricultural Institute of Slovenia, Hacquetova ulica 17, SI-1000 Ljubljana, Slovenia, PhD, *corresponding author: helena.basa@kis.si
} 
For determining the PPP residues in strawberries, a number of analytical methods were published. The first step in the methods is usually performed by liquidliquid extraction, with three main solvents being used: ethylacetate (Berrada et al., 2006; Ferrer et al., 2005), acetonitrile -also known as the QuEChERS method (Bakirci et al., 2014; Lehotay et al., 2007) or acetone (Jardim et al., 2012; Stan, 2000). Our laboratory used acetone because of its high volatility and miscibility with the water present in strawberry matrices. For the better extraction of active substance residues, we added dichloromethane and petroleum ether to the acetone. In this way, a wide range of active substances from medium polar (e.g. diazinon and dimethoate) to nonpolar (e.g. chlorpyrifos and cyhalothrin-lambda) were extracted. The extraction of PPP residues from the strawberry matrix is complicated because of its acidity. Therefore, in our laboratory, $\mathrm{pH}$ adjustment was used for better extraction efficiency, similar to the in QuEChERS method. $\mathrm{CH}_{3} \mathrm{COONa}$ and acetic acid were added to the strawberry matrix, which enhanced the extraction efficiency of $\mathrm{pH}$ sensitive active compounds (e.g. pirimicarb and pyrimethanil).

For determining active substance residues, chromatography is usually used. Gas chromatographs
(GC), used for non-polar to medium polar and volatile compounds, can be connected to a flame ionisation detector (FID), electron capture detector (ECD), nitrogen phosphor detector (NPD), flame photometric detector (FPD) or mass spectrometer (MS). In our laboratory, an MS was used as this is the only system that enables unequivocal qualitative and quantitative detection of active substance residues based on chromatographic retention time and mass spectra.

The purpose of this paper is to present the introduced, modified ( $\mathrm{pH}$ adjustment) and then validated gas chromatography-mass spectrometry (GC-MS) method, which enables the qualitative and quantitative determination of a wide range of active compounds in strawberries and their residues in one chromatographic run. Statistical analyses for the obtained data were used: for linearity using the $\mathrm{F}$ test, for accuracy by checking recoveries and cooperation in interlaboratory comparisons, for precision according to ISO 5725 standard and for measurement uncertainty by multiplying the standard deviation by Student's t factor for 9 degrees of freedom and a $95 \%$ confidence level. Finally, method implementation in practice was performed.

\section{MATERIALS AND METHODS}

\subsection{Materials}

Chemicals:

Acetone (Merck), dichloromethane (Merck), ethyl acetate (Merck), cyclohexane (Merck) and petroleum ether (Merck) with p.a. grade and GC grade were used as solvents in our experiment. Similarly, only active substances (dr. Ehrenstorfer, Pestanal) with the highest available purity on the market (a minimum of $95 \%$ ) were used.

Preparation of the solutions:

Stock solutions in a mixture of ethyl acetate and cyclohexane in a ratio of 1 to 1 of the individual active substances were prepared in $25 \mathrm{ml}$ volumetric flasks with concentrations of $625 \mu \mathrm{g}$ pesticide $\mathrm{ml}^{-1}$. From 53 stock solutions, two mixed solutions of all 53 active substances were prepared in $500 \mathrm{ml}$ volumetric flasks: one at a concentration of $5 \mu \mathrm{g} \mathrm{ml}^{-1}$ and the other at the limit of quantification (LOQ) of the active substances. All the solutions used to determine the linearity and LOQs were prepared from the $5 \mu \mathrm{g} \mathrm{ml}^{-1}$ mixed solution with proper dilutions. For other validation parameters, both mixed solutions $\left(5 \mu \mathrm{g} \mathrm{ml}^{-1}\right.$ concentration and the concentration at LOQ) were used. For standard solutions, solvents of GC grade were used.

\subsection{Procedure}

To $20 \mathrm{~g}$ of homogenised blank matrix (milled strawberries, which contain no PPP residues) or homogenised sample, $2 \mathrm{~g}$ of anhydrous $\mathrm{CH}_{3} \mathrm{COONa}$ was added. Afterwards $40 \mathrm{ml}$ of acetone p.a. and $0.4 \mathrm{ml}$ $100 \%$ acetic acid were added. The mixture was homogenised for 2 minutes with mixer (Ultra-turrax $\mathrm{T}$ 25, Ika-Werke). Then $80 \mathrm{ml}$ mixture of petroleum ether p.a. and dichloromethane p.a. at a ratio of 1:1 was added and mixed for another 2 minutes with a mixer. This mixture was filtered into the separatory funnel, containing $3 \mathrm{~g}$ of $\mathrm{NaCl}$. The vessel was rinsed with 80 $\mathrm{ml}$ of a mixture of petroleum ether p.a. and dichloromethane p.a. at a ratio of 1:1 (v/v). The solvent was added to the separatory funnel, which was shaken for 1 minute. The upper organic phase was filtered through $15 \mathrm{~g}$ anhydrous $\mathrm{Na}_{2} \mathrm{SO} 4$ in $500 \mathrm{ml}$ Soxhlet flask. The lower water phase was re-extracted twice using the same procedure. Solvents were evaporated to approximately $2 \mathrm{ml}$ on a rotavapor and dried with a nitrogen flow.

$8 \mathrm{ml}$ of a mixture of cyclohexane p.a. and ethyl acetate p.a. at a ratio $1: 1(\mathrm{v} / \mathrm{v})$ were added to dry extract. After filtration through a $0.2 \mu \mathrm{m}$ pore size filter, $5 \mathrm{ml}$ of the extract was cleaned using a gel permeation 
chromatograph, containing a column filled with biobeds SX3. The flow of the mobile phase (ethyl acetate p.a. and cyclohexane p.a., v/v = 1:1) through the GPC column was $5 \mathrm{ml} \mathrm{min}^{-1}$. The 90-200 $\mathrm{ml}$ of the eluate was collected into a Soxhlet flask, evaporated to approximately $2 \mathrm{ml}$ on a rotavapor and dried with a nitrogen flow. To the dry eluate, $2 \mathrm{ml}$ of the mixture of

ethyl acetate p.a. and cyclohexane p.a. at a ratio of $1: 1$ $(\mathrm{v} / \mathrm{v})$ was added in case of sample preparation. In the case of the matrix match standards, $2 \mathrm{ml}$ of the working solutions with proper concentrations were added.

\subsection{Determination}

Table 1: Chromatographic conditions of the GC (HP 6890)-MS (HP 5973) system:

\begin{tabular}{|c|c|}
\hline Liner: & HP 5181-3316 \\
\hline Temperature of injector: & $250^{\circ} \mathrm{C}$ \\
\hline Injection type: & Pulsed Splitless \\
\hline Precolumn: & $2 \mathrm{~m} * 0,25 \mathrm{~mm}$ \\
\hline Column: & HP $5 \mathrm{MS}, 30 \mathrm{~m}^{*} 0.25 \mathrm{~mm}, 0.25 \mu \mathrm{m}$ film \\
\hline Temperature gradient of column: & $\begin{array}{l}55^{\circ} \mathrm{C} 2 \mathrm{~min} \\
55^{\circ} \mathrm{C}-130{ }^{\circ} \mathrm{C} 25^{\circ} \mathrm{C} / \mathrm{min} \\
130{ }^{\circ} \mathrm{C} 1 \mathrm{~min} \\
130^{\circ} \mathrm{C}-180{ }^{\circ} \mathrm{C} 5^{\circ} \mathrm{C} / \mathrm{min} \\
180^{\circ} \mathrm{C} 30 \mathrm{~min} \\
180^{\circ} \mathrm{C}-230{ }^{\circ} \mathrm{C} 20{ }^{\circ} \mathrm{C} / \mathrm{min} \\
230^{\circ} \mathrm{C} 16 \mathrm{~min} \\
230^{\circ} \mathrm{C}-250{ }^{\circ} \mathrm{C} 20{ }^{\circ} \mathrm{C} / \mathrm{min} \\
250^{\circ} \mathrm{C} 13 \mathrm{~min} \\
250^{\circ} \mathrm{C}-280^{\circ} \mathrm{C} 20{ }^{\circ} \mathrm{C} / \mathrm{min} \\
280^{\circ} \mathrm{C} 20 \mathrm{~min}\end{array}$ \\
\hline Temperature of ion source: & $230^{\circ} \mathrm{C}$ \\
\hline Temperature of connector: & $280^{\circ} \mathrm{C}$ \\
\hline Temperature of detector: & $150^{\circ} \mathrm{C}$ \\
\hline Carrier gas: & Helium $6.0,1.2 \mathrm{ml} / \mathrm{min}$ constant flow \\
\hline Volume of injection: & $1 \mu \mathrm{l}$ \\
\hline
\end{tabular}

Table 2: Detection (selective ion monitoring):

\begin{tabular}{|c|c|}
\hline active substance & $\mathbf{T}, \mathrm{Q}_{1}, \mathrm{Q}_{2}, \mathrm{Q} 3(\mathbf{m} / \mathbf{z})$ \\
\hline aldrin & 263, 265, 261 \\
\hline azinphos-methyl & $\mathbf{1 6 0}, 132,105$ \\
\hline azoxystrobin & 344, 388, 345 \\
\hline bifenthrin & $\mathbf{1 8 1}, 165,166$ \\
\hline bromopropylate & $\mathbf{1 8 3}, 341,185$ \\
\hline bupirimate & 273, 316, 208 \\
\hline captan & 79, $107,119,149$ \\
\hline chlorothalonil & 266, 264, 268 \\
\hline chlorpropham & 213, 127, 154 \\
\hline chlorpyriphos & $\mathbf{3 1 4}, 316,197$ \\
\hline chlorpyriphos-methyl & 286, 288, 125 \\
\hline cyhalotrin- $\lambda$ & 181, 197, 208 \\
\hline cypermethrin (four isomers) & $\mathbf{1 8 1}, 163,165$ \\
\hline cyprodinil & $\mathbf{2 2 4}, 225,210$ \\
\hline \multirow[t]{4}{*}{ DDT (5 isomers) } & DDD-o,p: 235, 237, 165 \\
\hline & DDD-p,p and DDT-o,p: 235, 237, 165 \\
\hline & DDE-p,p: 318, 246, 248 \\
\hline & DDT-p,p: 235, 237, 165 \\
\hline
\end{tabular}


Helena BAŠA ČESNIK

\begin{tabular}{|c|c|}
\hline active substance & $\mathbf{T}, \mathrm{Q}_{1}, \mathrm{Q}_{2}, \mathrm{Q} 3(\mathbf{m} / \mathbf{z})$ \\
\hline deltamethrin & $\mathbf{1 8 1}, 251,255$ \\
\hline diazinon & 179, 304, 199 \\
\hline dichlofluanid & 226, 123,167 \\
\hline dimethoate & $\mathbf{8 7}, 229,143$ \\
\hline diphenylamine & 169, 167,168 \\
\hline endrin & $263,261,265$ \\
\hline fenitrothion & 277, 260, 109 \\
\hline fenthion & 278, 279, 280 \\
\hline fludioxonil & 248, 154, 127 \\
\hline folpet & $260,262,130$ \\
\hline HCH-alpha & 219, 181,183 \\
\hline heptachlor & $272,274,270$ \\
\hline heptenophos & $\mathbf{1 2 4}, 215,250$ \\
\hline iprodione & 314, 316,187 \\
\hline kresoxim-methyl & 116, 206, 131 \\
\hline lindane & $\mathbf{1 8 3}, 219,181$ \\
\hline mecarbam & 131, 159, 329 \\
\hline metalaxyl+metalaxyl-M & 249, 206, 234 \\
\hline methidathion & $145,85,125$ \\
\hline myclobutanil & $\mathbf{1 7 9}, 288,150$ \\
\hline parathion & 291, 292, 235 \\
\hline penconazole & 248, 159,161 \\
\hline permethrin (2 isomers) & $\mathbf{1 8 3}, 163,165$ \\
\hline phosalone & $\mathbf{1 8 2}, 367,121$ \\
\hline pirimicarb & 166, 238, 167 \\
\hline pirimiphos-methyl & $290,305,276$ \\
\hline propyzamide & 173, 175,145 \\
\hline pyridaphenthion & $199,340,188$ \\
\hline pyrimethanil & 198, 199, 200 \\
\hline quinalphos & 146, 298, 157 \\
\hline spiroxamine (2 isomers) & 100, 126, 198 \\
\hline tolclofos-methyl & $\mathbf{2 6 5}, 267,250$ \\
\hline tolylfluanid & 238, 137,240 \\
\hline triadimefon & 208, 210,181 \\
\hline triadimenol (2 isomers) & $\mathbf{1 1 2}, 168,128$ \\
\hline triazophos & 161, 162, 285 \\
\hline trifloxystrobin & 116, 222, 186 \\
\hline vinclozolin & 285, 124,187 \\
\hline
\end{tabular}

\subsection{Sampling}

Strawberry samples were randomly taken in May and June 2007 directly in the field after the expiration of pre-harvest interval. Samples originated from 6 production areas in Slovenia: Celje, Kranj, Ljubljana, Maribor, Murska Sobota and Novo mesto. 


\section{RESULTS AND DISCUSSION}

The previous protocol for the determination of PPP residues in fruit and vegetables was published before (Baša Česnik et al., 2006). The disadvantage of this procedure was, that when it was used for strawberries, some active substances were not extracted at all. Recoveries of previous procedure were compared to recoveries of new procedure (the one that includes $\mathrm{pH}$ adjustment) for two parallel samples of blank strawberries (strawberries that contained no PPP residues) spiked at level $0.2 \mathrm{mg} \mathrm{kg}^{-1}$. The new procedure differs from old procedure only in step where the anhydrous $\mathrm{CH}_{3} \mathrm{COONa}$ and the $100 \%$ acetic acid are added to the sample. $\mathrm{pH}$ adjustment enabled extraction of bupimirate, pirimicarb, pyrimethanil and spiroxamine, where recoveries were 0 without $\mathrm{pH}$ adjustment.

\subsection{Linearity and limits of quantification}

Linearity was verified using the matrix match standards (five repetitions for one concentration level, three to seven concentration levels for the calibration curve). The linearity and range were determined by linear regression using the $\mathrm{F}$ test. The linear model is fit and remains linear throughout the range presented in Table 1. The limits of quantification (LOQs) were estimated from chromatograms of the matrix match standards. LOQs were chosen at $\mathrm{S} / \mathrm{N}=10$. The $\mathrm{LOQ}$ is the lowest value of the linearity range for particular active substance presented in Table 3 .

Table 3: Linearity

\begin{tabular}{|c|c|c|c|c|c|}
\hline active substance & $\begin{array}{l}\text { linearity } \\
\text { range } \\
\left(\mathrm{mg} \mathrm{kg}^{-1}\right)\end{array}$ & $\mathrm{R}^{2}$ & active substance & $\begin{array}{l}\text { linearity } \\
\text { range } \\
\left(\mathrm{mg} \mathrm{kg}^{-1}\right) \\
\end{array}$ & $\mathrm{R}^{2}$ \\
\hline aldrin & $0.005-0.2$ & 0.997 & heptenophos & $0.01-0.2$ & 0.997 \\
\hline azinphos-methyl & $0.01-0.2$ & 0.989 & iprodione & $0.01-0.2$ & 0.995 \\
\hline azoxystrobin & $0.04-0.2$ & 0.985 & kresoxim-methyl & $0.02-0.2$ & 0.995 \\
\hline bifenthrin & $0.01-0.2$ & 0.997 & lindane & $0.01-0.2$ & 0.997 \\
\hline bromopropylate & $0.01-0.2$ & 0.997 & mecarbam & $0.04-0.2$ & 0.995 \\
\hline bupirimate & $0.02-0.2$ & 0.995 & metalaxyl+metalaxyl-M & $0.01-0.2$ & 0.998 \\
\hline captan & $0.1-0.2$ & 0.994 & methidathion & $0.01-0.2$ & 0.995 \\
\hline chlorothalonil & $0.01-0.2$ & 0.995 & myclobutanil & $0.05-0.2$ & 0.996 \\
\hline chlorpropham & $0.01-0.2$ & 0.997 & parathion & $0.03-1.0$ & 0.992 \\
\hline chlorpyriphos & $0.01-0.2$ & 0.997 & penconazole & $0.01-0.2$ & 0.996 \\
\hline chlorpyriphos-methyl & $0.02-0.2$ & 0.997 & permethrin & $0.02-0.2$ & 0.994 \\
\hline cyhalotrin-lambda & $0.01-0.5$ & 0.977 & phosalone & $0.01-0.2$ & 0.993 \\
\hline cypermethrin & $0.03-0.2$ & 0.991 & pirimicarb & $0.01-0.2$ & 0.997 \\
\hline cyprodinil & $0.01-0.2$ & 0.996 & pirimiphos-methyl & $0.01-0.2$ & 0.998 \\
\hline DDT & $0.05-1.0$ & 0.997 & propyzamide & $0.01-0.2$ & 0.997 \\
\hline deltamethrin & $0.03-0.2$ & 0.989 & pyridaphenthion & $0.01-1.0$ & 0.991 \\
\hline diazinon & $0.01-0.2$ & 0.998 & pyrimethanil & $0.01-0.2$ & 0.997 \\
\hline dichlofluanid & $0.01-0.2$ & 0.997 & quinalphos & $0.01-0.2$ & 0.996 \\
\hline
\end{tabular}


Helena BAŠA ČESNIK

\begin{tabular}{|l|r|r|l|r|r|}
\hline \multicolumn{1}{|c|}{ active substance } & $\begin{array}{c}\text { linearity } \\
\text { range } \\
\left(\mathrm{mg} \mathrm{kg}^{-1}\right)\end{array}$ & \multicolumn{1}{|c|}{$\mathrm{R}^{2}$} & \multicolumn{1}{|c|}{ active substance } & $\begin{array}{c}\text { linearity } \\
\text { range } \\
\left(\mathrm{mg} \mathrm{kg}^{-1}\right)\end{array}$ & $\mathrm{R}^{2}$ \\
\hline dimethoate & $0.01-0.2$ & 0.995 & spiroxamine & $0.02-1.0$ & 0.993 \\
\hline diphenylamine & $0.01-0.2$ & 0.996 & tolclofos-methyl & $0.01-0.2$ & 0.997 \\
\hline endrin & $0.01-0.2$ & 0.996 & tolylfluanid & $0.01-0.2$ & 0.996 \\
\hline fenitrothion & $0.01-1.0$ & 0.991 & triadimefon & $0.02-0.2$ & 0.997 \\
\hline fenthion & $0.005-0.2$ & 0.996 & triadimenol & $0.02-0.2$ & 0.994 \\
\hline fludioxonil & $0.01-0.2$ & 0.992 & triazophos & 0.2 & 0.992 \\
\hline folpet & $0.02-1.0$ & 0.988 & trifloxystrobin & $0.03-0.2$ & 0.996 \\
\hline HCH-alpha & $0.005-0.2$ & 0.997 & vinclozolin & $0.01-0.2$ & 0.997 \\
\hline heptachlor & $0.005-0.2$ & 0.998 & & & \\
\hline
\end{tabular}

\subsection{Accuracy}

Accuracy was verified by checking the recoveries. Ten extracts of spiked blank strawberry homogenate (milled strawberries that contained no PPP residues) were prepared for each spiking level in the shortest period possible. Each extract was injected twice. The average of the recoveries was calculated. According to the requirements for the method validation procedures (Document N ${ }^{\circ}$ SANTE/11945/2015), acceptable mean recoveries are those within the range of 70-120\%, with an associated repeatability $\mathrm{RSD}_{\mathrm{r}} \leq 20 \%$. Our recoveries of the spiking level at LOQ ranged from $96.6 \%$ to $105.4 \%$ with $\mathrm{RSD}_{\mathrm{r}} \leq 15 \%$, except for $\mathrm{HCH}$-alpha were the $\mathrm{RSD}_{\mathrm{r}}$ was $23 \%$. At spiking level $0.2 \mathrm{mg} \mathrm{kg}^{-1}$,

Table 4: Recoveries for spiked strawberry blank matrix

\begin{tabular}{|l|r|r|r|r|r|r|}
\hline active substance & $\begin{array}{c}\text { spiking level } \\
\left(\mathrm{mg} \mathrm{kg}^{-1}\right)\end{array}$ & \multicolumn{1}{c|}{$\begin{array}{c}\text { recovery } \\
(\%)\end{array}$} & $\begin{array}{c}\text { RSD } \\
(\%)\end{array}$ & $\begin{array}{c}\text { spiking level } \\
\left(\mathrm{mg} \mathrm{kg}^{-1}\right)\end{array}$ & $\begin{array}{c}\text { recovery } \\
(\%)\end{array}$ & $\begin{array}{c}\text { RSD } \\
(\%)\end{array}$ \\
\hline aldrin & 0.005 & 99.1 & 6.8 & 0.2 & 97.7 & 7.8 \\
\hline azinphos-methyl & 0.01 & 98.9 & 11.4 & 0.2 & 99.9 & 12.2 \\
\hline azoxystrobin & 0.04 & 98.8 & 14.5 & 0.2 & 99.8 & 12.2 \\
\hline bifenthrin & 0.01 & 101.0 & 12.1 & 0.2 & 97.7 & 9.2 \\
\hline bromopropylate & 0.01 & 101.3 & 13.8 & 0.2 & 97.6 & 9.3 \\
\hline bupirimate & 0.02 & 103.2 & 13.6 & 0.2 & 97.5 & 9.6 \\
\hline captan & 0.1 & 101.5 & 9.9 & 0.2 & 97.4 & 9.4 \\
\hline chlorothalonil & 0.01 & 96.6 & 9.3 & 0.2 & 97.8 & 9.2 \\
\hline chlorpropham & 0.01 & 100.6 & 8.6 & 0.2 & 97.6 & 8.2 \\
\hline chlorpyriphos & 0.01 & 102.6 & 12.4 & 0.2 & 97.1 & 8.0 \\
\hline chlorpyriphos-methyl & 0.02 & 102.1 & 9.6 & 0.2 & 97.5 & 7.8 \\
\hline cyhalotrin-lambda & 0.01 & 99.8 & 8.4 & 0.2 & 97.3 & 10.6 \\
\hline
\end{tabular}

recoveries ranged from $96.8 \%$ to $99.9 \%$ with $\mathrm{RSD}_{\mathrm{r}} \leq$ $13 \%$.

According to the guidelines for single-laboratory validation (Alder et al., 2000), the acceptable mean recoveries:

- at level $>0.1 \mathrm{mg} \mathrm{kg}^{-1} \leq 1 \mathrm{mg} \mathrm{kg}^{-1}$ are within the range $70-110 \%$, with an associated repeatability RSDr $\leq$ $15 \%$,

- at level $>0.01 \mathrm{mg} \mathrm{kg}^{-1} \leq 0.1 \mathrm{mg} \mathrm{kg}^{-1}$ are within the range $70-120 \%$, with an associated repeatability RSDr $\leq 20 \%$ and

- at level $>0.001 \mathrm{mg} \mathrm{kg}^{-1} \leq 0.01 \mathrm{mg} \mathrm{kg}^{-1}$ are within the range $60-120 \%$, with an associated repeatability RSDr $\leq 30 \%$.

These requirements were achieved for all 53 active compounds. The results are given in Table 4. 
Validation of the multiresidual GC-MS method for determining plant protection product residues in strawberries

\begin{tabular}{|c|c|c|c|c|c|c|}
\hline active substance & $\begin{array}{c}\text { spiking level } \\
\left(\mathrm{mg} \mathrm{kg}^{-1}\right)\end{array}$ & $\begin{array}{c}\text { recovery } \\
(\%)\end{array}$ & $\begin{array}{c}\mathrm{RSD} \\
(\%)\end{array}$ & $\begin{array}{c}\text { spiking level } \\
\left(\mathrm{mg} \mathrm{kg}^{-1}\right)\end{array}$ & $\begin{array}{c}\text { recovery } \\
(\%)\end{array}$ & $\begin{array}{l}\text { RSD } \\
(\%)\end{array}$ \\
\hline cypermethrin & 0.03 & 97.3 & 6.9 & 0.2 & 98.8 & 12.5 \\
\hline cyprodinil & 0.01 & 103.1 & 12.1 & 0.2 & 97.5 & 9.3 \\
\hline DDT & 0.05 & 101.6 & 9.8 & 1.0 & 97.4 & 9.1 \\
\hline deltamethrin & 0.03 & 99.9 & 10.4 & 0.2 & 98.9 & 12.4 \\
\hline diazinon & 0.01 & 104.2 & 12.0 & 0.2 & 97.8 & 7.4 \\
\hline dichlofluanid & 0.01 & 100.1 & 8.8 & 0.2 & 97.4 & 8.1 \\
\hline dimethoate & 0.01 & 102.7 & 10.9 & 0.2 & 97.9 & 8.9 \\
\hline diphenylamine & 0.01 & 99.5 & 7.3 & 0.2 & 98.0 & 7.5 \\
\hline endrin & 0.01 & 97.9 & 9.2 & 0.2 & 97.5 & 8.7 \\
\hline fenitrothion & 0.01 & 100.1 & 8.3 & 0.2 & 97.0 & 10.2 \\
\hline fenthion & 0.005 & 101.8 & 13.9 & 0.2 & 97.4 & 8.3 \\
\hline fludioxonil & 0.01 & 99.3 & 11.8 & 0.2 & 99.3 & 11.3 \\
\hline folpet & 0.02 & 101.7 & 11.2 & 0.2 & 97.6 & 10.7 \\
\hline HCH-alpha & 0.005 & 100.9 & 23.0 & 0.2 & 97.8 & 7.5 \\
\hline heptachlor & 0.005 & 99.8 & 7.0 & 0.2 & 97.9 & 7.5 \\
\hline heptenophos & 0.01 & 101.2 & 8.4 & 0.2 & 97.9 & 7.9 \\
\hline iprodione & 0.01 & 99.1 & 11.6 & 0.2 & 98.2 & 10.3 \\
\hline kresoxim-methyl & 0.02 & 103.3 & 12.2 & 0.2 & 97.5 & 9.6 \\
\hline lindane & 0.01 & 99.4 & 8.4 & 0.2 & 97.9 & 7.4 \\
\hline mecarbam & 0.04 & 103.1 & 11.0 & 0.2 & 97.7 & 8.8 \\
\hline metalaxyl+metalaxyl-M & 0.01 & 103.2 & 11.1 & 0.2 & 97.6 & 8.1 \\
\hline methidathion & 0.01 & 103.5 & 12.0 & 0.2 & 98.0 & 9.8 \\
\hline myclobutanil & 0.05 & 104.5 & 14.6 & 0.2 & 97.8 & 9.7 \\
\hline parathion & 0.03 & 98.3 & 7.7 & 0.2 & 96.8 & 10.1 \\
\hline penconazole & 0.01 & 104.9 & 10.2 & 0.2 & 97.7 & 9.1 \\
\hline permethrin & 0.02 & 100.3 & 12.6 & 0.2 & 98.0 & 11.3 \\
\hline phosalone & 0.01 & 101.3 & 11.5 & 0.2 & 98.1 & 10.9 \\
\hline pirimicarb & 0.01 & 101.5 & 10.8 & 0.2 & 97.8 & 8.0 \\
\hline pirimiphos-methyl & 0.01 & 103.6 & 12.1 & 0.2 & 97.9 & 8.2 \\
\hline propyzamide & 0.01 & 102.1 & 9.0 & 0.2 & 97.4 & 8.4 \\
\hline pyridaphenthion & 0.01 & 103.6 & 10.7 & 0.2 & 97.8 & 11.0 \\
\hline pyrimethanil & 0.01 & 100.8 & 9.4 & 0.2 & 97.6 & 8.2 \\
\hline quinalphos & 0.01 & 104.5 & 13.7 & 0.2 & 97.3 & 9.3 \\
\hline spiroxamine & 0.03 & 102.2 & 10.9 & 0.2 & 97.4 & 8.1 \\
\hline tolclofos-methyl & 0.01 & 101.6 & 8.3 & 0.2 & 97.8 & 7.8 \\
\hline tolylfluanid & 0.01 & 100.1 & 9.7 & 0.2 & 97.2 & 8.7 \\
\hline triadimefon & 0.02 & 101.5 & 10.6 & 0.2 & 97.3 & 8.8 \\
\hline triadimenol & 0.02 & 105.4 & 10.6 & 0.2 & 97.6 & 9.9 \\
\hline triazophos & 0.01 & 102.1 & 12.4 & 0.2 & 97.6 & 11.5 \\
\hline trifloxystrobin & 0.03 & 102.9 & 13.4 & 0.2 & 97.8 & 9.9 \\
\hline vinclozolin & 0.01 & 100.3 & 8.6 & 0.2 & 97.5 & 8.6 \\
\hline
\end{tabular}


Helena BAŠA ČESNIK

Table 5: Interlaboratory comparison results (in $\mathrm{mg} \mathrm{kg}^{-1}$ ) (BIPEA, 2015)

\begin{tabular}{|l|r|r|r|r|r|r|}
\hline \multicolumn{1}{|c|}{ active substance } & \multicolumn{1}{c|}{ reference } & \multicolumn{1}{c|}{ tolerance } & \multicolumn{1}{c|}{ maximum } & \multicolumn{1}{c|}{ minimum } & \multicolumn{1}{c|}{ our result } & \multicolumn{1}{l|}{$\mathrm{Z}$} \\
\hline azoxystrobin & 0.053 & 0.027 & 0.080 & 0.026 & 0.056 & 0.22 \\
\hline bifenthrin & 0.022 & 0.011 & 0.033 & 0.011 & 0.018 & -0.73 \\
\hline cyhalotrin-lambda & 0.064 & 0.032 & 0.096 & 0.032 & 0.062 & -0.13 \\
\hline deltamethrin & 0.166 & 0.076 & 0.242 & 0.090 & 0.164 & -0.05 \\
\hline diphenylamine & 0.129 & 0.062 & 0.191 & 0.067 & 0.112 & -0.55 \\
\hline dimethoate & 0.066 & 0.033 & 0.099 & 0.033 & 0.061 & -0.3 \\
\hline fenitrothion & 0.044 & 0.022 & 0.066 & 0.022 & 0.050 & 0.55 \\
\hline phosalone & 0.163 & 0.075 & 0.238 & 0.088 & 0.158 & -0.13 \\
\hline kresoxim-methyl & 0.023 & 0.012 & 0.035 & 0.011 & 0.020 & -0.5 \\
\hline lindane & 0.146 & 0.068 & 0.214 & 0.078 & 0.140 & -0.18 \\
\hline metalaxyl+metalaxyl-M & 0.036 & 0.018 & 0.054 & 0.018 & 0.028 & -0.89 \\
\hline myclobutanil & 0.032 & 0.016 & 0.048 & 0.016 & 0.031 & -0.13 \\
\hline pirimicarb & 0.169 & 0.078 & 0.247 & 0.091 & 0.152 & -0.44 \\
\hline
\end{tabular}

Accuracy was also checked with participation in a proficiency testing scheme organised by BIPEA (Bureau interprofessionnel d'études analytiques). All the results were within the required range $(-2 \geq z \leq 2)$. The results are presented in Table 3.

\subsection{Precision}

For the determination of precision (ISO 5725), i.e. repeatability and reproducibility, the extracts of spiked blank strawberry matrix were analysed at two concentration levels. Within the period of 10 days, two parallel extracts were prepared each day for each concentration level. Each one was injected once. Then the standard deviation of repeatability of the level and the standard deviation of reproducibility of the level were both calculated. The results are given in Table 6 . 
Validation of the multiresidual GC-MS method for determining plant protection product residues in strawberries

Table 6: Standard deviation of repeatability $\left(\mathrm{s}_{\mathrm{r}}\right)$ and reproducibility $\left(\mathrm{s}_{\mathrm{R}}\right)$ of the method

\begin{tabular}{|c|c|c|c|c|c|c|c|c|}
\hline active substance & $\begin{array}{l}\text { spiking level } \\
\left(\mathrm{mg} \mathrm{kg}^{-1}\right)\end{array}$ & $\begin{array}{l}\text { means of the } \\
\text { levels } \\
\left(\mathrm{mg} \mathrm{kg}^{-1}\right)\end{array}$ & $\begin{array}{l}\mathrm{S}_{\mathrm{r}} \\
\left(\mathrm{mg} \mathrm{kg}^{-1}\right)\end{array}$ & $\begin{array}{l}\mathrm{S}_{\mathrm{R}} \\
\left(\mathrm{mg} \mathrm{kg}^{-1}\right)\end{array}$ & $\begin{array}{l}\text { spiking level } \\
\left(\mathrm{mg} \mathrm{kg}^{-1}\right)\end{array}$ & $\begin{array}{l}\text { means of the } \\
\text { levels (mg kg } \\
\left.{ }^{1}\right)\end{array}$ & $\begin{array}{l}\mathrm{S}_{\mathrm{r}} \\
\left(\mathrm{mg} \mathrm{kg}^{-1}\right)\end{array}$ & $\begin{array}{l}\mathrm{S}_{\mathrm{R}} \\
\left(\mathrm{mg} \mathrm{kg}^{-1}\right)\end{array}$ \\
\hline aldrin & 0.005 & 0.0050 & 0.0002 & 0.0003 & 0.2 & 0.19 & 0.01 & 0.01 \\
\hline azinphos-methyl & 0.01 & 0.010 & 0.001 & 0.001 & 0.2 & 0.19 & 0.02 & 0.02 \\
\hline azoxystrobin & 0.04 & 0.038 & 0.005 & 0.006 & 0.2 & 0.19 & 0.02 & 0.02 \\
\hline bifenthrin & 0.01 & 0.0098 & 0.0007 & 0.0008 & 0.2 & 0.19 & 0.01 & 0.01 \\
\hline bromopropylate & 0.01 & 0.0097 & 0.0009 & 0.0009 & 0.2 & 0.19 & 0.01 & 0.01 \\
\hline bupirimate & 0.02 & 0.020 & 0.001 & 0.001 & 0.2 & 0.19 & 0.01 & 0.01 \\
\hline captan & 0.1 & 0.10 & 0.02 & 0.02 & 0.2 & 0.19 & 0.01 & 0.02 \\
\hline chlorothalonil & 0.01 & 0.0099 & 0.0007 & 0.0007 & 0.2 & 0.19 & 0.01 & 0.01 \\
\hline chlorpropham & 0.01 & 0.0098 & 0.0005 & 0.0006 & 0.2 & 0.19 & 0.01 & 0.01 \\
\hline chlorpyriphos & 0.01 & 0.0098 & 0.0005 & 0.0007 & 0.2 & 0.19 & 0.01 & 0.01 \\
\hline chlorpyriphos-methyl & 0.02 & 0.020 & 0.001 & 0.001 & 0.2 & 0.19 & 0.01 & 0.01 \\
\hline cyhalotrin-lambda & 0.01 & 0.0095 & 0.0007 & 0.0009 & 0.2 & 0.19 & 0.01 & 0.01 \\
\hline cypermethrin & 0.03 & 0.029 & 0.003 & 0.003 & 0.2 & 0.19 & 0.01 & 0.01 \\
\hline cyprodinil & 0.01 & 0.0098 & 0.0006 & 0.0007 & 0.2 & 0.19 & 0.01 & 0.01 \\
\hline DDT & 0.05 & 0.050 & 0.003 & 0.004 & 1.0 & 0.95 & 0.05 & 0.06 \\
\hline deltamethrin & 0.03 & 0.029 & 0.003 & 0.003 & 0.2 & 0.19 & 0.02 & 0.02 \\
\hline diazinon & 0.01 & 0.0098 & 0.0005 & 0.0006 & 0.2 & 0.19 & 0.01 & 0.01 \\
\hline dichlofluanid & 0.01 & 0.0096 & 0.0006 & 0.0009 & 0.2 & 0.19 & 0.01 & 0.01 \\
\hline dimethoate & 0.01 & 0.0097 & 0.0007 & 0.0008 & 0.2 & 0.19 & 0.01 & 0.01 \\
\hline diphenylamine & 0.01 & 0.0099 & 0.0004 & 0.0005 & 0.2 & 0.19 & 0.01 & 0.01 \\
\hline endrin & 0.01 & 0.0100 & 0.0006 & 0.0006 & 0.2 & 0.19 & 0.01 & 0.01 \\
\hline fenitrothion & 0.01 & 0.0098 & 0.0007 & 0.0008 & 0.2 & 0.19 & 0.01 & 0.01 \\
\hline fenthion & 0.005 & 0.0049 & 0.0003 & 0.0004 & 0.2 & 0.19 & 0.01 & 0.01 \\
\hline fludioxonil & 0.01 & 0.010 & 0.001 & 0.001 & 0.2 & 0.19 & 0.01 & 0.02 \\
\hline folpet & 0.02 & 0.020 & 0.004 & 0.004 & 0.2 & 0.19 & 0.01 & 0.02 \\
\hline HCH-alpha & 0.005 & 0.0049 & 0.0002 & 0.0002 & 0.2 & 0.19 & 0.01 & 0.01 \\
\hline heptachlor & 0.005 & 0.0050 & 0.0003 & 0.0003 & 0.2 & 0.19 & 0.01 & 0.01 \\
\hline
\end{tabular}


Helena BAŠA ČESNIK

\begin{tabular}{|c|c|c|c|c|c|c|c|c|}
\hline active substance & $\begin{array}{l}\text { spiking level } \\
\left(\mathrm{mg} \mathrm{kg}^{-1}\right)\end{array}$ & $\begin{array}{l}\text { means of the } \\
\text { levels } \\
\left(\mathrm{mg} \mathrm{kg}^{-1}\right)\end{array}$ & $\begin{array}{l}\mathrm{S}_{\mathrm{r}} \\
\left(\mathrm{mg} \mathrm{kg}^{-1}\right)\end{array}$ & $\begin{array}{l}\mathrm{S}_{\mathrm{R}} \\
\left(\mathrm{mg} \mathrm{kg}^{-1}\right)\end{array}$ & $\begin{array}{l}\text { spiking level } \\
\left(\mathrm{mg} \mathrm{kg}^{-1}\right)\end{array}$ & $\begin{array}{l}\text { means of the } \\
\text { levels }(\mathrm{mg} \mathrm{kg} \\
{ }^{1} \text { ) }\end{array}$ & $\begin{array}{l}\mathrm{S}_{\mathrm{r}} \\
\left(\mathrm{mg} \mathrm{kg}^{-1}\right)\end{array}$ & $\begin{array}{l}\mathrm{S}_{\mathrm{R}} \\
\left(\mathrm{mg} \mathrm{kg}^{-1}\right)\end{array}$ \\
\hline heptenophos & 0.01 & 0.0098 & 0.0004 & 0.0006 & 0.2 & 0.19 & 0.01 & 0.01 \\
\hline iprodione & 0.01 & 0.0097 & 0.0009 & 0.0011 & 0.2 & 0.19 & 0.01 & 0.01 \\
\hline kresoxim-methyl & 0.02 & 0.020 & 0.001 & 0.001 & 0.2 & 0.19 & 0.01 & 0.01 \\
\hline lindane & 0.01 & 0.0100 & 0.0005 & 0.0005 & 0.2 & 0.19 & 0.01 & 0.01 \\
\hline mecarbam & 0.04 & 0.039 & 0.003 & 0.003 & 0.2 & 0.19 & 0.01 & 0.01 \\
\hline metalaxyl+metalaxyl-M & 0.01 & 0.0098 & 0.0004 & 0.0004 & 0.2 & 0.19 & 0.01 & 0.01 \\
\hline methidathion & 0.01 & 0.0098 & 0.0009 & 0.0009 & 0.2 & 0.19 & 0.01 & 0.01 \\
\hline myclobutanil & 0.05 & 0.049 & 0.004 & 0.004 & 0.2 & 0.19 & 0.01 & 0.01 \\
\hline parathion & 0.03 & 0.029 & 0.002 & 0.002 & 0.2 & 0.19 & 0.01 & 0.01 \\
\hline penconazole & 0.01 & 0.0097 & 0.0006 & 0.0007 & 0.2 & 0.19 & 0.01 & 0.01 \\
\hline permethrin & 0.02 & 0.020 & 0.002 & 0.002 & 0.2 & 0.19 & 0.01 & 0.01 \\
\hline phosalone & 0.01 & 0.0097 & 0.0009 & 0.0011 & 0.2 & 0.19 & 0.01 & 0.01 \\
\hline pirimicarb & 0.01 & 0.0099 & 0.0006 & 0.0006 & 0.2 & 0.19 & 0.01 & 0.01 \\
\hline pirimiphos-methyl & 0.01 & 0.0098 & 0.0005 & 0.0006 & 0.2 & 0.19 & 0.01 & 0.01 \\
\hline propyzamide & 0.01 & 0.0098 & 0.0005 & 0.0005 & 0.2 & 0.19 & 0.01 & 0.01 \\
\hline pyridaphenthion & 0.01 & 0.010 & 0.001 & 0.001 & 0.2 & 0.19 & 0.01 & 0.01 \\
\hline pyrimethanil & 0.01 & 0.0098 & 0.0006 & 0.0006 & 0.2 & 0.19 & 0.01 & 0.01 \\
\hline quinalphos & 0.01 & 0.0098 & 0.0007 & 0.0009 & 0.2 & 0.19 & 0.01 & 0.01 \\
\hline spiroxamine & 0.03 & 0.0296 & 0.001 & 0.002 & 0.2 & 0.19 & 0.01 & 0.01 \\
\hline tolclofos-methyl & 0.01 & 0.0099 & 0.0005 & 0.0005 & 0.2 & 0.19 & 0.01 & 0.01 \\
\hline tolylfluanid & 0.01 & 0.010 & 0.001 & 0.001 & 0.2 & 0.19 & 0.01 & 0.01 \\
\hline triadimefon & 0.02 & 0.020 & 0.001 & 0.001 & 0.2 & 0.19 & 0.01 & 0.01 \\
\hline triadimenol & 0.02 & 0.0195 & 0.002 & 0.002 & 0.2 & 0.19 & 0.01 & 0.01 \\
\hline triazophos & 0.01 & 0.0097 & 0.0008 & 0.0009 & 0.2 & 0.19 & 0.01 & 0.01 \\
\hline trifloxystrobin & 0.03 & 0.029 & 0.002 & 0.003 & 0.2 & 0.19 & 0.01 & 0.01 \\
\hline vinclozolin & 0.01 & 0.0099 & 0.0006 & 0.0006 & 0.2 & 0.19 & 0.01 & 0.01 \\
\hline
\end{tabular}




\subsection{Uncertainty of repeatability and uncertainty of reproducibility}

Uncertainty of repeatability and uncertainty of reproducibility were calculated by multiplying the standard deviation of repeatability and the standard deviation of reproducibility by Student's $t$ factor for 9 degrees of freedom and a $95 \%$ confidence level $\left(t_{95 ; 9}=\right.$ 2.262).

$\mathrm{Ur}=\mathrm{t}_{95 ; 9} \times \mathrm{S}_{\mathrm{r}} ; \mathrm{UR}=\mathrm{t}_{95 ; 9} \mathrm{XS}_{\mathrm{R}}$

The results are presented in Table 7. The measurement uncertainty for PPP residues is set in the Official Gazette of the Republic of Slovenia (Republic of Slovenia, 2007). Its value is $50 \%$. With validation, analysts must prove that their measurement uncertainty is below or equal to the official measurement uncertainty.

\subsection{Sample analysis}

The method was checked in practice. 19 strawberry samples were analysed for the presence of all 53 validated active substances. 10 samples, which represent $52.6 \%$ of all the analysed samples contained no residues. 5 active substances, all fungicides, were found: chlorothalonil, cyprodinil, fludioxonil, metalaxyl+metalaxyl-M and pyrimethanil. Other active substances were below the LOQ. The most frequently measured was cyprodinil, which was found in 8 samples, representing $42.1 \%$ of all the analysed samples. The reason is probably that this substance is included in the PPP Switch 62.5 WG, which is the mixture of fungicides cyprodinil and fludioxonil used for strawberries and sold in Slovenia. 9 samples, which represent $47.4 \%$ of all the analysed samples contained PPP residues in the range $0.01-0.44 \mathrm{mg} / \mathrm{kg}$. Multiple residues ( 2 or more active substances) were found in 5 samples, representing $26.3 \%$ of all the analysed samples. None of the substances exceeded the valid MRL. Therefore, the conclusion was drawn that farmers were using PPPs according to good agriculture practice described on the labels of the PPPs. Also, these strawberries presented no risk to consumers. The results are presented in Table 8.

Comparing our results with the literature we observed that PPP residues in strawberries in Slovenia are mainly comparable to observations of other authors. Jardim et al. (2012) found pesticide residues in Brazilia in $76.3 \%$ of strawberry samples; $71.6 \%$ of them had multiple residues and $13.5 \%$ of them were exceeding the MRL. In Slovenia, the amount of positive samples was about $29 \%$ lower, the amount of multiple residues was about $45 \%$ lower and no MRL exceedances were observed. On the other hand Poulsen et al. (2017) reported that in Denmark, $37 \%$ of the analysed samples contained multiple residues, which is approximately $11 \%$ higher than in Slovenia.

In strawberry samples in Poland, Szpyrka et al. (2015) found cypermethrin, deltamethrin and trifloxystrobin among the active substances that we both analysed. On the other hand, again in strawberry samples in Poland, Sójka et al. (2015) found the fungicides cyprodinil (mean content $0.16 \mathrm{mg} \mathrm{kg}^{-1}$ ), fludioxonil (mean content $0.115 \mathrm{mg} \mathrm{kg}^{-1}$ ) and pyrimethanil (mean content 0.056 $\mathrm{mg} \mathrm{kg}{ }^{-1}$ ), as well as the insecticide chlorpyrifos (mean content $0.012 \mathrm{mg} \mathrm{kg}^{-1}$ ) among the active substances that we both analysed. The mean contents of cyprodinil and fludioxonil were comparable to ours, while the content of pyrimethanil was slightly lower. Chlorpyriphos was not found in our research. In protected strawberries Allen et al. (2015) found cyprodinil (mean content $0.062 \mathrm{mg} \mathrm{kg}^{-1}$ ) and iprodione (mean content $0.055 \mathrm{mg}$ $\mathrm{kg}^{-1}$ ) among the active substances that we both analysed. The cyprodinil mean content was in the range of contents that we measured, while iprodione was not found in our research. 
Helena BAŠA ČESNIK

Table 7: Uncertainty of repeatability $\left(U_{r}\right)$ and reproducibility $\left(U_{R}\right)$ of the method

\begin{tabular}{|c|c|c|c|c|c|c|c|c|c|c|}
\hline active substance & $\begin{array}{l}\text { spiking level } \\
\left(\mathrm{mg} \mathrm{kg}^{-1}\right)\end{array}$ & $\begin{array}{l}\mathrm{U}_{\mathrm{r}} \\
\left(\mathrm{mg} \mathrm{kg}^{-1}\right)\end{array}$ & $\begin{array}{l}\mathrm{U}_{\mathrm{r}} \\
(\%)\end{array}$ & $\begin{array}{l}\mathrm{U}_{\mathrm{R}} \\
\left(\mathrm{mg} \mathrm{kg}^{-1}\right)\end{array}$ & $\begin{array}{l}\mathrm{U}_{\mathrm{R}} \\
(\%) \\
\end{array}$ & $\begin{array}{l}\text { spiking level } \\
\left(\mathrm{mg} \mathrm{kg}^{-1}\right)\end{array}$ & $\begin{array}{l}\mathrm{U}_{\mathrm{r}} \\
\left(\mathrm{mg} \mathrm{kg}^{-1}\right)\end{array}$ & $\begin{array}{l}\mathrm{U}_{\mathrm{r}} \\
(\%) \\
\end{array}$ & $\begin{array}{l}\mathrm{U}_{\mathrm{R}} \\
\left(\mathrm{mg} \mathrm{kg}^{-1}\right)\end{array}$ & $\begin{array}{l}\mathrm{U}_{\mathrm{R}} \\
(\%) \\
\end{array}$ \\
\hline aldrin & 0.005 & 0.0006 & 12.0 & 0.0006 & 12.0 & 0.2 & 0.02 & 10.0 & 0.02 & 10.0 \\
\hline azinphos-methyl & 0.01 & 0.003 & 30.0 & 0.003 & 30.0 & 0.2 & 0.04 & 20.0 & 0.05 & 25.0 \\
\hline azoxystrobin & 0.04 & 0.01 & 25.0 & 0.01 & 25.0 & 0.2 & 0.04 & 20.0 & 0.04 & 20.0 \\
\hline bifenthrin & 0.01 & 0.002 & 20.0 & 0.002 & 20.0 & 0.2 & 0.02 & 10.0 & 0.03 & 15.0 \\
\hline bromopropylate & 0.01 & 0.002 & 20.0 & 0.002 & 20.0 & 0.2 & 0.02 & 10.0 & 0.03 & 15.0 \\
\hline bupirimate & 0.02 & 0.003 & 15.0 & 0.003 & 15.0 & 0.2 & 0.03 & 15.0 & 0.03 & 15.0 \\
\hline captan & 0.1 & 0.04 & 40.0 & 0.04 & 40.0 & 0.2 & 0.04 & 20.0 & 0.1 & 50.0 \\
\hline chlorothalonil & 0.01 & 0.002 & 20.0 & 0.002 & 20.0 & 0.2 & 0.03 & 15.0 & 0.03 & 15.0 \\
\hline chlorpropham & 0.01 & 0.001 & 10.0 & 0.001 & 10.0 & 0.2 & 0.02 & 10.0 & 0.02 & 10.0 \\
\hline chlorpyriphos & 0.01 & 0.001 & 10.0 & 0.002 & 20.0 & 0.2 & 0.02 & 10.0 & 0.02 & 10.0 \\
\hline chlorpyriphos-methyl & 0.02 & 0.003 & 15.0 & 0.003 & 15.0 & 0.2 & 0.02 & 10.0 & 0.03 & 15.0 \\
\hline cyhalotrin-lambda & 0.01 & 0.002 & 20.0 & 0.002 & 20.0 & 0.2 & 0.03 & 15.0 & 0.03 & 15.0 \\
\hline cypermethrin & 0.03 & 0.007 & 23.3 & 0.007 & 23.3 & 0.2 & 0.03 & 15.0 & 0.03 & 15.0 \\
\hline cyprodinil & 0.01 & 0.001 & 10.0 & 0.002 & 20.0 & 0.2 & 0.02 & 10.0 & 0.03 & 15.0 \\
\hline DDT & 0.05 & 0.007 & 14.0 & 0.008 & 16.0 & 1.0 & 0.12 & 12.0 & 0.14 & 14.0 \\
\hline deltamethrin & 0.03 & 0.006 & 20.0 & 0.006 & 20.0 & 0.2 & 0.04 & 20.0 & 0.04 & 20.0 \\
\hline diazinon & 0.01 & 0.001 & 10.0 & 0.001 & 10.0 & 0.2 & 0.02 & 10.0 & 0.02 & 10.0 \\
\hline dichlofluanid & 0.01 & 0.001 & 10.0 & 0.002 & 20.0 & 0.2 & 0.03 & 15.0 & 0.02 & 10.0 \\
\hline dimethoate & 0.01 & 0.002 & 20.0 & 0.002 & 20.0 & 0.2 & 0.03 & 15.0 & 0.03 & 15.0 \\
\hline diphenylamine & 0.01 & 0.0009 & 9.0 & 0.0011 & 11.0 & 0.2 & 0.02 & 10.0 & 0.02 & 10.0 \\
\hline endrin & 0.01 & 0.001 & 10.0 & 0.001 & 10.0 & 0.2 & 0.02 & 10.0 & 0.03 & 15.0 \\
\hline fenitrothion & 0.01 & 0.002 & 20.0 & 0.002 & 20.0 & 0.2 & 0.03 & 15.0 & 0.03 & 15.0 \\
\hline fenthion & 0.005 & 0.0007 & 14.0 & 0.0008 & 16.0 & 0.2 & 0.02 & 10.0 & 0.02 & 10.0 \\
\hline fludioxonil & 0.01 & 0.002 & 20.0 & 0.003 & 30.0 & 0.2 & 0.03 & 15.0 & 0.04 & 20.0 \\
\hline folpet & 0.02 & 0.009 & 45.0 & 0.009 & 45.0 & 0.2 & 0.03 & 15.0 & 0.03 & 15.0 \\
\hline HCH-alpha & 0.005 & 0.0005 & 10.0 & 0.0005 & 10.0 & 0.2 & 0.02 & 10.0 & 0.02 & 10.0 \\
\hline heptachlor & 0.005 & 0.0006 & 12.0 & 0.0006 & 12.0 & 0.2 & 0.02 & 10.0 & 0.02 & 10.0 \\
\hline
\end{tabular}


Validation of the multiresidual GC-MS method for determining plant protection product residues in strawberries

\begin{tabular}{|c|c|c|c|c|c|c|c|c|c|c|}
\hline active substance & $\begin{array}{l}\text { spiking level } \\
\left(\mathrm{mg} \mathrm{kg}^{-1}\right)\end{array}$ & $\begin{array}{l}\mathrm{U}_{\mathrm{r}} \\
\left(\mathrm{mg} \mathrm{kg}^{-1}\right)\end{array}$ & $\begin{array}{l}\mathrm{U}_{\mathrm{r}} \\
(\%) \\
\end{array}$ & $\begin{array}{l}\mathrm{U}_{\mathrm{R}} \\
\left(\mathrm{mg} \mathrm{kg}^{-1}\right)\end{array}$ & $\begin{array}{l}\mathrm{U}_{\mathrm{R}} \\
(\%) \\
\end{array}$ & $\begin{array}{l}\text { spiking level } \\
\left(\mathrm{mg} \mathrm{kg}^{-1}\right)\end{array}$ & $\begin{array}{l}\mathrm{U}_{\mathrm{r}} \\
\left(\mathrm{mg} \mathrm{kg}^{-1}\right)\end{array}$ & $\begin{array}{l}\mathrm{U}_{\mathrm{r}} \\
(\%) \\
\end{array}$ & $\begin{array}{l}\mathrm{U}_{\mathrm{R}} \\
\left(\mathrm{mg} \mathrm{kg}^{-1}\right)\end{array}$ & $\begin{array}{l}\mathrm{U}_{\mathrm{R}} \\
(\%)\end{array}$ \\
\hline heptenophos & 0.01 & 0.001 & 10.0 & 0.001 & 10.0 & 0.2 & 0.02 & 10.0 & 0.03 & 15.0 \\
\hline iprodione & 0.01 & 0.002 & 20.0 & 0.002 & 20.0 & 0.2 & 0.03 & 15.0 & 0.03 & 15.0 \\
\hline kresoxim-methyl & 0.02 & 0.003 & 15.0 & 0.003 & 15.0 & 0.2 & 0.03 & 15.0 & 0.03 & 15.0 \\
\hline lindane & 0.01 & 0.001 & 10.0 & 0.001 & 10.0 & 0.2 & 0.02 & 10.0 & 0.02 & 10.0 \\
\hline mecarbam & 0.04 & 0.007 & 17.5 & 0.007 & 17.5 & 0.2 & 0.02 & 10.0 & 0.03 & 15.0 \\
\hline metalaxyl+metalaxyl-M & 0.01 & 0.0009 & 9.0 & 0.0010 & 10.0 & 0.2 & 0.02 & 10.0 & 0.03 & 15.0 \\
\hline methidathion & 0.01 & 0.002 & 20.0 & 0.002 & 20.0 & 0.2 & 0.03 & 15.0 & 0.03 & 15.0 \\
\hline myclobutanil & 0.05 & 0.009 & 18.0 & 0.009 & 18.0 & 0.2 & 0.02 & 10.0 & 0.03 & 15.0 \\
\hline parathion & 0.03 & 0.005 & 16.7 & 0.005 & 16.7 & 0.2 & 0.03 & 15.0 & 0.03 & 15.0 \\
\hline penconazole & 0.01 & 0.001 & 10.0 & 0.002 & 20.0 & 0.2 & 0.02 & 10.0 & 0.03 & 15.0 \\
\hline permethrin & 0.02 & 0.004 & 20.0 & 0.005 & 25.0 & 0.2 & 0.03 & 15.0 & 0.03 & 15.0 \\
\hline phosalone & 0.01 & 0.002 & 20.0 & 0.002 & 20.0 & 0.2 & 0.03 & 15.0 & 0.03 & 15.0 \\
\hline pirimicarb & 0.01 & 0.001 & 10.0 & 0.001 & 10.0 & 0.2 & 0.03 & 15.0 & 0.03 & 15.0 \\
\hline pirimiphos-methyl & 0.01 & 0.001 & 10.0 & 0.001 & 10.0 & 0.2 & 0.02 & 10.0 & 0.02 & 10.0 \\
\hline propyzamide & 0.01 & 0.001 & 10.0 & 0.001 & 10.0 & 0.2 & 0.02 & 10.0 & 0.03 & 15.0 \\
\hline pyridaphenthion & 0.01 & 0.002 & 20.0 & 0.003 & 30.0 & 0.2 & 0.03 & 15.0 & 0.03 & 15.0 \\
\hline pyrimethanil & 0.01 & 0.001 & 10.0 & 0.001 & 10.0 & 0.2 & 0.02 & 10.0 & 0.03 & 15.0 \\
\hline quinalphos & 0.01 & 0.002 & 20.0 & 0.002 & 20.0 & 0.2 & 0.02 & 10.0 & 0.02 & 10.0 \\
\hline spiroxamine & 0.03 & 0.003 & 10.0 & 0.004 & 13.3 & 0.2 & 0.02 & 10.0 & 0.02 & 10.0 \\
\hline tolclofos-methyl & 0.01 & 0.001 & 10.0 & 0.001 & 10.0 & 0.2 & 0.02 & 10.0 & 0.02 & 10.0 \\
\hline tolylfluanid & 0.01 & 0.002 & 20.0 & 0.002 & 20.0 & 0.2 & 0.02 & 10.0 & 0.03 & 15.0 \\
\hline triadimefon & 0.02 & 0.003 & 15.0 & 0.003 & 15.0 & 0.2 & 0.02 & 10.0 & 0.03 & 15.0 \\
\hline triadimenol & 0.02 & 0.003 & 15.0 & 0.004 & 20.0 & 0.2 & 0.02 & 10.0 & 0.03 & 15.0 \\
\hline triazophos & 0.01 & 0.002 & 20.0 & 0.002 & 20.0 & 0.2 & 0.03 & 15.0 & 0.03 & 15.0 \\
\hline trifloxystrobin & 0.03 & 0.005 & 16.7 & 0.006 & 20.0 & 0.2 & 0.03 & 15.0 & 0.03 & 15.0 \\
\hline vinclozolin & 0.01 & 0.001 & 10.0 & 0.001 & 10.0 & 0.2 & 0.02 & 10.0 & 0.02 & 10.0 \\
\hline
\end{tabular}


Table 8: Contents of active substances found in 19 strawberry samples

\begin{tabular}{|c|c|c|c|c|c|}
\hline & $\begin{array}{l}\text { chlorothalonil } \\
\left(\mathrm{mg} \mathrm{kg}^{-1}\right)\end{array}$ & $\begin{array}{l}\text { cyprodinil } \\
\left(\mathrm{mg} \mathrm{kg}^{-1}\right)\end{array}$ & $\begin{array}{l}\text { fludioxonil } \\
\left(\mathrm{mg} \mathrm{kg}^{-1}\right)\end{array}$ & $\begin{array}{l}\text { metalaxyl+metalaxyl-M } \\
\left(\mathrm{mg} \mathrm{kg}^{-1}\right)\end{array}$ & $\begin{array}{l}\text { pyrimethanil } \\
\left(\mathrm{mg} \mathrm{kg}^{-1}\right)\end{array}$ \\
\hline $\operatorname{MRL}\left(\mathrm{mg} \mathrm{kg}^{-1}\right)$ & 4.0 & 5.0 & 4.0 & 0.6 & 5.0 \\
\hline \multicolumn{6}{|l|}{ sample no. } \\
\hline 1 & - & - & - & - & - \\
\hline 2 & - & - & - & - & - \\
\hline 3 & - & 0.04 & - & - & - \\
\hline 4 & - & 0.04 & - & - & - \\
\hline 5 & - & - & - & - & - \\
\hline 6 & - & - & - & - & - \\
\hline 7 & - & - & - & - & - \\
\hline 8 & 0.01 & - & - & - & - \\
\hline 9 & - & - & - & - & - \\
\hline 10 & - & 0.02 & - & - & - \\
\hline 11 & 0.10 & 0.02 & - & - & - \\
\hline 12 & - & - & - & - & - \\
\hline 13 & - & 0.24 & 0.17 & - & 0.13 \\
\hline 14 & 0.06 & 0.02 & - & - & - \\
\hline 15 & - & - & - & - & - \\
\hline 16 & - & 0.24 & - & 0.02 & - \\
\hline 17 & 0.02 & 0.08 & - & - & 0.44 \\
\hline 18 & - & - & - & - & - \\
\hline 19 & - & - & - & - & - \\
\hline
\end{tabular}

- means <LOQ

MRL is maximum residue level

\section{CONCLUSIONS}

According to the validation, the method is suitable for the determination of at least 53 active compounds and their residues in strawberries. The method could be expanded to more active substances. The system is linear with an $\mathrm{R}^{2}$ higher or equal than 0.977 . The LOQs range from $0.005 \mathrm{mg} \mathrm{kg}^{-1}$ for aldrin to $0.1 \mathrm{mg} \mathrm{kg}^{-1}$ for captan. Recoveries range from $96.6 \%$ (chlorothalonil) to $105.4 \%$ (triadimenol) at a spiking level equal to the LOQ. Uncertainty of reproducibility ranges from $10 \%$ for vinclozolin to $50 \%$ for captan. The method is fit for purpose and is accredited according to the SIST EN ISO/IEC 17025 standard by the Slovenian accreditation body SA.

\section{ACKNOWLEDGEMENT}

The author would like to thank Mateja Fortuna and Danijela Cvijin for help with extract preparation. The author acknowledges the financial support of the
Slovenian Research Agency (research core funding No. P4-0133). 


\section{REFERENCES}

Alder L., Hill A., Holland P.T.,.Lantos J, Lee S.M., MacNeil J.D., O'Rangers J., van Zoonen P., Ambrus A. (2000). Guidelines for single-laboratory validation of analytical methods for trace-level concentrations of organic chemicals, Principles and practices of method validation (ed.: A. Fajgelj, A. Ambrus). The Royal Society of Chemistry, pp. 179 -252 .

Allen G., Halsall C.J., Ukpebor J., Paul N.D., Ridall G., Wargent J.J. (2015). Increased occurrence of pesticide residues on crops grown in protected environments compared to crops grown in open field conditions. Chemosphere, 119, 1428-1435, doi.org/10.1016/j.chemosphere.2014.10.066

Bakirci G.T., Acay D.B.Y., Bakirci F., Ötleş S. (2014). Pesticide residues in fruits and vegetables from the Aegean region, Turkey. Food Chemistry, 160, 379392, doi.org/10.1016/j.foodchem.2014.02.051

Baša Česnik H., Gregorčič A., Velikonja Bolta Š., Kmecl V. (2006). Monitoring of pesticide residues in apples, lettuce and potato of the Slovene origin, 2001-04. Food Additives and Contaminants, 23,164-173, doi.org/10.1080/02652030500401199

Baša Česnik H., Velikonja Bolta Š., Gregorčič A. (2009). Pesticide Residues in Agricultural Products of the Slovene Origin Found in 2007. Acta Chimica Slovenica, 56, 484-493.

Berrada H., Fernández M., Rulz M.J., Mólto J.C., Mañes J. (2006). Exposure assessment of fruits contaminated with pesticide residues from Valencia, 2001-03. Food Additives and Contaminants, 23, 674-682, doi.org/10.1080/02652030600599132

BIPEA. (2015). Bureau interprofessionnel d'études analytiques. Laboratory comparisons report, Strawberry, 05-2319. June 2015. Gienevilliers, BIPEA.

Document $N^{\circ}$ SANTE/11945/2015. Analytical Quality Control and Method Validation Procedures for pesticide Residues Analysis in Food and Feed. DG SANTE, European Comission, 2015.

Ferrer I., García-Reyes J.F., Mezcua M., Thurman E.M., Fernández-Alba A.R. (2005). Multi-residue pesticide analysis in fruits and vegetables by liquid chromatography-time-of-flight mass spectrometry. Journal of Chromatography A, 1082, 81-90, doi.org/10.1016/j.chroma.2005.03.040

ISO 5725. (1994). Accuracy (trueness and precision) of measurement methods and results - Part2: Basic method for the determination of repeatability and reproducibility of a standard measurement method, pp. 1-42.

Jardim A.N.O., Caldas E.D. (2012). Brazilian monitoring programs for pesticide residues in foodResults from 2001 to 2010. Food Control, 25, 607616, doi: 10.1016/j.food.cont.2011.11.001.

Poulsen M.E., Andersen J.H., Petersen A., Jensen B.H. (2017). Results from the Danish monitoring programme for pesticide residues from the period 2004-2011. Food Control, 74, 25-33, doi.org/10.1016/j.foodcont.2016.11.022

Republic of Slovenia. (2007). Rules on Amendments to the Rules on the Restructuring of Pesticides in or on Foodstuffs and Agricultural Products, Official Gazette of the Republic of Slovenia No. 108, 27.11.2007, page 14834 .

Sójka M., Miszczak A., Sikorski P., Zagibajlo K., Karlińska E., Kosmala M. (2015). Pesticide residue levels in strawberry processing by-products that are rich in ellagitannins and an assessment of the dietary risk to consumers. NFS Journal, 1, 31-37, doi.org/10.1016/j.nfs.2015.09.001

Stan H.J. (2000). Pesticide residue analysis in foodstuffs applying capillary gas chromatography with mass spectrometric detection: State-of-the-art use of modified DFG-multimethod S19 and automated data evaluation. Journal of Chromatography A, 892, 347-377, doi.org/10.1016/S00219673(00)00308-3

Szpyrka E., Kurdziel A., Matyaszek A., Podbielska M., Rupar J., Slowik-Borowiec M. (2015). Evaluation of pesticide residues in fruits and vegetables from the region of south-eastern Poland. Food Control, 48 , 137-142, doi.org/10.1016/j.foodcont.2014.05.039. 